\title{
The Banach-Mazur Distance to the Cube in Low Dimensions
}

\author{
Steven Taschuk
}

Received: 6 November 2009 / Revised: 28 January 2010 / Accepted: 29 January 2010 /

Published online: 18 February 2010

(C) Springer Science+Business Media, LLC 2010

\begin{abstract}
We show that the Banach-Mazur distance from any centrally symmetric convex body in $\mathbb{R}^{n}$ to the $n$-dimensional cube is at most

$$
\sqrt{n^{2}-2 n+2+\frac{2}{\sqrt{n+2}-1}},
$$

which improves previously known estimates for "small" $n \geq 3$. (For large $n$, asymptotically better bounds are known; in the asymmetric case, exact bounds are known.) The proof of our estimate uses an idea of Lassak and the existence of two nearly orthogonal contact points in John's decomposition of the identity. Our estimate on such contact points is closely connected to a well-known estimate of Gerzon on equiangular systems of lines.
\end{abstract}

Keywords Convex bodies · Banach-Mazur distance · Equiangular lines · John's theorem

\section{Introduction}

In this note, a convex body is a convex compact set with nonempty interior. A set $A \subseteq \mathbb{R}^{n}$ is called symmetric if $A=-A$. The Banach-Mazur distance between two symmetric convex bodies $K$ and $L$ in $\mathbb{R}^{n}$ is

$$
d_{\mathrm{BM}}(K, L)=\inf \left\{\lambda>0: K \subseteq T L \subseteq \lambda K \text { for some } T \in \mathrm{GL}\left(\mathbb{R}^{n}\right)\right\} .
$$

The author was partially supported by an Alexander Graham Bell Canada Graduate Scholarship and by a Vanier Canada Graduate Scholarship, both from the Natural Sciences and Engineering Research Council of Canada. The author also thanks his supervisor Alexander Litvak for his support and advice and Márton Naszódi for his valuable comments on an early draft of this note.

S. Taschuk $(\bowtie)$

CAB 632, University of Alberta, Edmonton, AB T6G 2R3, Canada

e-mail: staschuk@ualberta.ca 
This distance is multiplicative: $d_{\mathrm{BM}}(K, M) \leq d_{\mathrm{BM}}(K, L) d_{\mathrm{BM}}(L, M)$.

The maximum Banach-Mazur distance between a symmetric convex body $K$ and the $n$-dimensional cube $B_{\infty}^{n}=[-1,1]^{n}$ is unknown for $n \geq 3$. (For $K$ that may be asymmetric, the maximum distance is exactly $n$; see Sect. 2.2 below.) The best previously known upper bound for small $n$ was due to Lassak [13], who showed that $d_{\mathrm{BM}}\left(K, B_{\infty}^{n}\right) \leq \sqrt{n^{2}-n+1}$ for any symmetric convex body $K$. Our main result, Theorem 1, is an improvement of Lassak's bound for all $n \geq 3$. (For example, for $n=3$, Lassak's bound gives $\sqrt{7}$, while Theorem 1 gives $\sqrt{6.618 \ldots}$.) Our bound is of interest only for small $n$ because, like Lassak's bound, it is asymptotically linear in $n$, but $\max _{K} d_{\mathrm{BM}}\left(K, B_{\infty}^{n}\right)$ is known to be of lower order; see Sect. 2.2.

Theorem 1 For any symmetric convex body $K$ in $\mathbb{R}^{n}, n \geq 3$,

$$
d_{\mathrm{BM}}\left(K, B_{\infty}^{n}\right) \leq \sqrt{n^{2}-2 n+2+\frac{2}{\sqrt{n+2}-1}} .
$$

The proof of Theorem 1 combines Lassak's idea with the following lemma on nearly orthogonal contact points in John configurations (a name we adopt to acknowledge their role in John's theorem, which is stated below as Theorem 6).

Definition 2 A finite collection $\left(u_{i}\right)_{1}^{m}$ of unit vectors in $\mathbb{R}^{n}$ is a John configuration if there exist positive real numbers $\left(c_{i}\right)_{1}^{m}$ such that

$$
\sum_{i} c_{i} u_{i} \otimes u_{i}=\mathrm{Id}
$$

where $u \otimes v$ denotes the map $\mathbb{R}^{n} \rightarrow \mathbb{R}^{n}, x \mapsto\langle u, x\rangle v$.

Lemma 3 If $\left(u_{i}\right)_{1}^{m}$ is a John configuration in $\mathbb{R}^{n}, n \geq 2$, then

$$
\min _{i, j}\left|\left\langle u_{i}, u_{j}\right\rangle\right| \leq \frac{1}{\sqrt{n+2}} .
$$

The case of equality in Lemma 3 is closely related to the question of how many lines there can be in an equiangular system of lines in $\mathbb{R}^{n}$. A theorem due to Gerzon (stated below as Theorem 7) asserts that such a system contains at most $\frac{1}{2} n(n+1)$ lines and gives necessary conditions on $n$ for this bound to be achieved (see Sect. 2.4 for details). The following proposition shows that Lemma 3 is sharp in $\mathbb{R}^{n}$ if and only if Gerzon's bound is sharp.

Proposition 4 Let $\left(u_{i}\right)_{1}^{m}$ be a finite collection of unit vectors in $\mathbb{R}^{n}, n \geq 2$. Then the following are equivalent:

(i) $\left(u_{i}\right)_{1}^{m}$ is a John configuration, and $\min _{i, j}\left|\left\langle u_{i}, u_{j}\right\rangle\right|=1 / \sqrt{n+2}$;

(ii) the lines spanned by the $u_{i}$ are equiangular, and $m=\frac{1}{2} n(n+1)$. 
Remark 5 In the proof of Proposition 4 given below, the direction (ii) $\Rightarrow$ (i) is obtained by considering the geometry of the set of operators $u_{i} \otimes u_{i}$ (under the HilbertSchmidt inner product), specifically by showing from condition (ii) that $\frac{1}{n}$ Id is their centroid. With a little more computation, one may actually show that when the conditions of Proposition 4 hold, these operators are the vertices of a regular simplex centered at $\frac{1}{n} \mathrm{Id}$, which accounts for the extremality of the situation.

\section{Background}

\subsection{Notation}

We work in $\mathbb{R}^{n}$ with its standard basis $\left(e_{i}\right)_{1}^{n}$, its canonical inner product $\langle\cdot, \cdot\rangle$, the associated Euclidean norm $|\cdot|$, and origin 0 . We write $B_{2}^{n}$ for the closed unit Euclidean ball and $B_{\infty}^{n}$ for the $n$-dimensional cube $[-1,1]^{n}$. The boundary of a set $A \subseteq \mathbb{R}^{n}$ is denoted $\partial A$; the convex hull of $A$ is denoted conv $A$; and the polar of $A$ is

$$
A^{\circ}=\left\{x \in \mathbb{R}^{n}:\langle x, a\rangle \leq 1 \text { for all } a \in A\right\} .
$$

\subsection{Distance to the Cube}

Asplund [1] showed that, for any symmetric convex body $K$ in the plane,

$$
d_{\mathrm{BM}}\left(K, B_{\infty}^{2}\right) \leq \frac{3}{2}
$$

with equality for the regular hexagon. See [11], §7, for a survey of this area in classical convex geometry. For general $n$, Lassak [13] showed that

$$
d_{\mathrm{BM}}\left(K, B_{\infty}^{n}\right) \leq \sqrt{n^{2}-n+1}
$$

The best known asymptotic estimates are

$$
c \sqrt{n} \log n \leq \max _{K} d_{\mathrm{BM}}\left(K, B_{\infty}^{n}\right) \leq C n^{5 / 6}
$$

for some absolute positive constants $c$ and $C$. The lower bound is due to Szarek [16]. The upper bound is the culmination of work by several authors: Bourgain and Szarek [5] obtained the first estimate of order $o(n)$; Szarek and Talagrand [17] proved $O\left(n^{7 / 8}\right)$; Giannopoulos [7] improved their argument to give $O\left(n^{5 / 6}\right)$. See [8], §7.2, for a survey of this area in asymptotic geometric analysis.

The analogous, more general, problem when $K$ is permitted to be an asymmetric convex body (and the notion of Banach-Mazur distance is suitably generalized) was solved by Gordon, Litvak, Meyer, and Pajor [10], who showed that $d_{\mathrm{BM}}(K, L) \leq n$ for any convex body $K$ and any symmetric convex body $L$ and that equality is attained if $K$ is the simplex. See [10], Theorem 5.5 and Corollary 5.8. 


\subsection{John Configurations}

Theorem 6 (John [12]) Let $K$ be a symmetric convex body in $\mathbb{R}^{n}$. Then $B_{2}^{n}$ is the ellipsoid of maximum volume in $K$ if and only if $B_{2}^{n} \subseteq K$ and $\partial B_{2}^{n} \cap \partial K$ contains a John configuration. Every symmetric convex body has one such affine image (up to orthogonal transformations).

The original paper of John is difficult to obtain; for proofs of Theorem 6, see, for example: [2], Lecture 3; [8], §2.3; [18], §15. John also proved a variant without the assumption of symmetry, which has been generalized to two arbitrary convex bodies; see $[3,9]$ and for the strongest form, [10], Theorem 3.8.

We will use the following well-known facts about John configurations. It is easy to show that the defining condition (1) is equivalent to the condition that

$$
\sum_{i} c_{i}\left\langle u_{i}, x\right\rangle^{2}=|x|^{2} \quad \text { for all } x \in \mathbb{R}^{n}
$$

Taking traces in (1) yields

$$
\sum_{i} c_{i}=n
$$

and so $\left(u_{i}\right)_{1}^{m}$ is a John configuration if and only if

$$
\frac{1}{n} \operatorname{Id} \in \operatorname{conv}\left\{u_{i} \otimes u_{i}: i=1, \ldots, m\right\} .
$$

Carathéodory's theorem (applied to the space of symmetric trace-one operators from $\mathbb{R}^{n}$ to $\mathbb{R}^{n}$ ) yields that any John configuration has a subset with at most $\frac{1}{2} n(n+1)$ elements which is also a John configuration.

If $K$ and $\left(u_{i}\right)_{1}^{m} \subseteq \partial B_{2}^{n} \cap \partial K$ are as in Theorem 6, then any halfspace that supports $K$ at $u_{i}$ also supports $B_{2}^{n}$ there, and so must be $\left\{u_{i}\right\}^{\circ}$; thus $K \subseteq\left\{ \pm u_{1}, \ldots, \pm u_{m}\right\}^{\circ}$. A straightforward computation using (3) and (4) shows that $\left\{ \pm u_{1}, \ldots, \pm u_{m}\right\}^{\circ} \subseteq$ $\sqrt{n} B_{2}^{n}$.

\subsection{Equiangular Lines}

Theorem 7 (Gerzon; [14], Theorem 3.5) An equiangular system of lines in $\mathbb{R}^{n}$ contains at most $\frac{1}{2} n(n+1)$ lines. If equality is achieved, then the angle between any pair of lines is $\arccos (1 / \sqrt{n+2})$, and moreover, either $n=2, n=3$, or $n+2$ is an odd square.

In fact, Gerzon's theorem as stated in [14] does not include the value of the angle, but the proof given there does establish it.

Systems of lines achieving Gerzon's bound are known to exist for the first few $n$ satisfying the conditions stated in Theorem 7, namely $n=2,3,7,23$; see [14]. The case of equality (or near-equality) in Gerzon's theorem is also of interest in the theory of frames; see [15] for a recent survey from this point of view. 


\section{Proofs}

Proof of Lemma 3 As noted in Sect. 2.3, we may assume that $m \leq \frac{1}{2} n(n+1)$. Let $\left(c_{i}\right)_{1}^{m}$ be positive real numbers such that $\sum_{i} c_{i} u_{i} \otimes u_{i}=$ Id. Let $X$ be a random variable in $\{1, \ldots, m\}$ with

$$
\operatorname{Prob}(X=j)=\frac{c_{j}}{n}
$$

Since

$$
\max _{i} c_{i} \geq \frac{1}{m} \sum_{i} c_{i}=\frac{n}{m} \geq \frac{2}{n+1},
$$

we have

$$
\begin{aligned}
\min _{i} \min _{j}\left\langle u_{i}, u_{j}\right\rangle^{2} & =\min _{i} \min _{j \neq i}\left\langle u_{i}, u_{j}\right\rangle^{2} \\
& \leq \min _{i} \mathbb{E}\left(\left\langle u_{i}, u_{X}\right\rangle^{2} \mid X \neq i\right)=\min _{i} \frac{1}{1-c_{i} / n} \sum_{j \neq i} \frac{c_{j}}{n}\left\langle u_{i}, u_{j}\right\rangle^{2} \\
& =\min _{i} \frac{1}{n-c_{i}}\left(\left|u_{i}\right|^{2}-c_{i}\left\langle u_{i}, u_{i}\right\rangle^{2}\right)=\min _{i} \frac{1-c_{i}}{n-c_{i}} \\
& \leq \frac{1-2 /(n+1)}{n-2 /(n+1)}=\frac{1}{n+2},
\end{aligned}
$$

as desired.

Remark 8 The idea of averaging over the $u_{i}$ according to their weights goes back to John [12], who used a similar argument to prove Jung's inequality [4], which asserts that if $r$ is the minimum radius of a sphere enclosing some set $A \subseteq \mathbb{R}^{d}$, then

$$
r \leq \sqrt{\frac{d}{2(d+1)}} \operatorname{diam} A,
$$

and equality is attained by regular simplices. In fact, one can deduce Lemma 3 from this inequality by taking $A$ to be the set of operators $u_{i} \otimes u_{i}$ (with geometry given by the Hilbert-Schmidt inner product, as in the proof of Proposition 4). The proof above, however, gives slightly more, since it shows that we may take $u_{i}$ to be any point with above-average weight; John's method cannot provide such information because he computes the average over all pairs $(i, j)$ with $i \neq j$ instead of fixing $i$ and averaging over $j$. (Compare also [15], Proposition 2, which uses a method resembling John's to estimate the maximum inner product between two vectors in a slightly different context.)

Remark 9 The weaker, though asymptotically equivalent, bound

$$
\min _{i, j}\left|\left\langle u_{i}, u_{j}\right\rangle\right| \leq \frac{1}{\sqrt{n}}
$$


is obtained in the first step of the proof of the classical Dvoretzky-Rogers lemma [6] (also [8], Theorem 2.3.3). The argument of Theorem 1 can be given using this bound, but the resulting bound on $d_{\mathrm{BM}}\left(K, B_{\infty}^{n}\right)$ does not improve Lassak's result (2) in the case $n=3$.

Proof of Theorem 1 Applying an affine transformation if necessary, we may assume that $B_{2}^{n}$ is the maximum volume ellipsoid of $K$. Let $\left(u_{i}\right)_{1}^{m}$ be a John configuration of contact points, as in Theorem 6. By Lemma 3, we may assume that

$$
\left|\left\langle u_{1}, u_{2}\right\rangle\right| \leq \frac{1}{\sqrt{n+2}}
$$

Let $V=\operatorname{span}\left\{u_{1}, u_{2}\right\}$. Rotating if necessary, we may assume that $V=\operatorname{span}\left\{e_{1}, e_{2}\right\}$ and $u_{1}+u_{2}$ is a multiple of $e_{1}+e_{2}$.

Let

$$
U=\left\{ \pm u_{1}, \pm u_{2}\right\}^{\circ}, \quad L=U \cap \sqrt{n} B_{2}^{n}, \quad \text { and } \quad M=U \cap \sqrt{n} B_{\infty}^{n} .
$$

We will show that

$$
d_{\mathrm{BM}}\left(K, B_{\infty}^{n}\right) \leq d_{\mathrm{BM}}(K, L) d_{\mathrm{BM}}(L, M) d_{\mathrm{BM}}\left(M, B_{\infty}^{n}\right) \leq \sqrt{n} \cdot \frac{r}{\sqrt{n}} \cdot 1=r,
$$

where $r=\max _{x \in M}|x|$.

To show that $d_{\mathrm{BM}}(K, L) \leq \sqrt{n}$, note that, by the remarks in Sect. 2.3, $K \subseteq L \subseteq$ $\sqrt{n} B_{2}^{n} \subseteq \sqrt{n} K$

To show that $d_{\mathrm{BM}}(L, M) \leq \frac{r}{\sqrt{n}}$, note first that $\sqrt{n} e_{3} \in M$, so $r \geq \sqrt{n}$. Thus $M \subseteq$ $\frac{r}{\sqrt{n}} U$. Furthermore, $M \subseteq r B_{2}^{n}=\frac{r}{\sqrt{n}} \sqrt{n} B_{2}^{n}$ by the definition of $r$. So $L \subseteq M \subseteq \frac{r}{\sqrt{n}} L$.

To show that $d_{\mathrm{BM}}\left(M, B_{\infty}^{n}\right)=1$, we will show that $M$ is a parallelotope. Consider a vertex $p$ of the rhombus $U \cap V$. (See Fig. 1.) Since $p$ is the intersection of the tangents to the circle $B_{2}^{n} \cap V$ at $u_{1}$ and $u_{2}$, the line $\ell$ joining $u_{1}$ and $u_{2}$ is $\partial\left(\{p\}^{\circ}\right)$, so

$$
\begin{aligned}
|p|^{2} & =\frac{1}{\operatorname{dist}(0, \ell)^{2}}=\frac{1}{\left|\frac{1}{2}\left(u_{1}+u_{2}\right)\right|^{2}}=\frac{2}{1+\left\langle u_{1}, u_{2}\right\rangle} \\
& \leq \frac{2}{1-\frac{1}{\sqrt{n+2}}}=2+\frac{2}{\sqrt{n+2}-1} .
\end{aligned}
$$

Fig. 1 The rhombus $U \cap V$ in the proof of Theorem 1

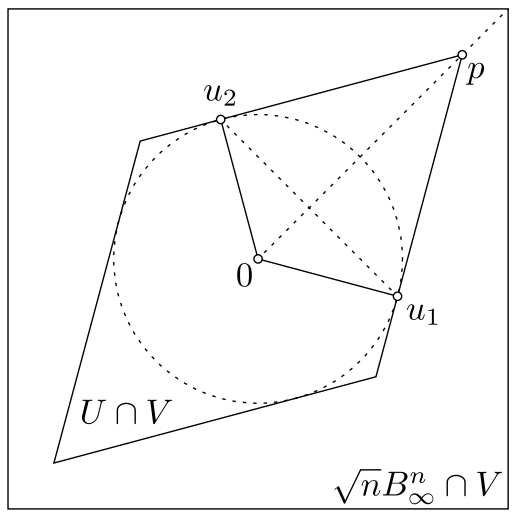


In particular, $|p|^{2}<4<2 n$, so the vertices of the rhombus $U \cap V$ are closer to the origin than the vertices of the square $\sqrt{n} B_{\infty}^{n} \cap V$ are. Recall that $u_{1}+u_{2}$ is a multiple of $e_{1}+e_{2}$, so the vertices of the rhombus lie on the same rays through the origin as the vertices of the square; thus,

$$
U \cap V \subseteq \sqrt{n} B_{\infty}^{n} \cap V
$$

as Fig. 1 shows. It then follows easily that

$$
M=U \cap \sqrt{n} B_{\infty}^{n}=U \cap V+\sqrt{n} B_{\infty}^{n} \cap V^{\perp},
$$

which is the sum of a rhombus and an $(n-2)$-dimensional cube in complementary subspaces, hence a parallelotope.

It remains only to estimate $r$. Let $x=v+w \in M$, where $v \in U \cap V$ and $w \in$ $\sqrt{n} B_{\infty}^{n} \cap V^{\perp}$. Then

$$
|x|^{2}=|v|^{2}+|w|^{2} \leq 2+\frac{2}{\sqrt{n+2}-1}+n(n-2) .
$$

Taking square roots yields the desired result.

Remark 10 Lassak's bound (2) is obtained by essentially the same method as in the above proof, namely truncating $B_{2}^{n}$ to obtain a body which more closely resembles $B_{\infty}^{n}$. The chief difference is that Lassak's argument uses only one contact point: in our notation, he uses $U=\left\{ \pm u_{1}\right\}^{\circ}$.

Proof of Proposition 4 Examining the inequalities in the proof of Lemma 3, we see that condition (i) holds if and only if:

1. $\left(u_{i}\right)$ is a John configuration with all weights equal;

2. $m=\frac{1}{2} n(n+1)$; and

3. all the values $\left\langle u_{i}, u_{j}\right\rangle^{2}$ with $i \neq j$ are equal.

The last two statements are exactly condition (ii), which thus clearly follows from condition (i).

Conversely, suppose that condition (ii) holds, so that $m=\frac{1}{2} n(n+1)$ and the points $\left(u_{i}\right)_{1}^{m}$ satisfy, say,

$$
\left\langle u_{i}, u_{j}\right\rangle^{2}= \begin{cases}1 & \text { if } i=j, \\ a & \text { if } i \neq j .\end{cases}
$$

We wish to show that the points $\left(u_{i}\right)_{1}^{m}$ are a John configuration with all weights equal, that is,

$$
\sum_{i} \frac{n}{m} u_{i} \otimes u_{i}=\mathrm{Id} .
$$

Consider the space of real symmetric $n \times n$ matrices, under the Hilbert-Schmidt inner product

$$
\langle\langle A, B\rangle\rangle=\operatorname{tr}(A B),
$$


and the associated norm $\|\cdot\|_{H S}$ (which in this finite-dimensional setting is sometimes called the Frobenius norm). With this inner product structure, the hyperplane $\{A: \operatorname{tr}(A)=1\}$ is at distance $1 / \sqrt{n}$ from the origin, and the (unique) closest point to the origin in this hyperplane is $\frac{1}{n}$ Id. On the other hand, $\operatorname{tr}\left(\frac{1}{m} \sum_{i} u_{i} \otimes u_{i}\right)=1$, and

$$
\begin{aligned}
\left\|\frac{1}{m} \sum_{i} u_{i} \otimes u_{i}\right\|_{\mathrm{HS}}^{2} & =\frac{1}{m^{2}} \sum_{i} \sum_{j}\left\langle\left\langle u_{i} \otimes u_{i}, u_{j} \otimes u_{j}\right\rangle\right\rangle=\frac{1}{m^{2}} \sum_{i} \sum_{j}\left\langle u_{i}, u_{j}\right\rangle^{2} \\
& =\frac{1}{m^{2}}\left(\sum_{i}\left|u_{i}\right|^{4}+\sum_{i} \sum_{j \neq i}\left\langle u_{i}, u_{j}\right\rangle^{2}\right)=\frac{1}{m}+\left(1-\frac{1}{m}\right) a=\frac{1}{n},
\end{aligned}
$$

since $m=\frac{1}{2} n(n+1)$ (by hypothesis) and $a=1 /(n+2)$ (by Theorem 7). Thus, $\frac{1}{m} \sum_{i} u_{i} \otimes u_{i}$ is also a closest point to the origin in the trace-one hyperplane, so by uniqueness, $\frac{1}{m} \sum_{i} u_{i} \otimes u_{i}=\frac{1}{n} \mathrm{Id}$, as desired.

\section{References}

1. Asplund, E.: Comparison between plane symmetric convex bodies and parallelograms. Math. Scand. 8, 171-180 (1960)

2. Ball, K.: An elementary introduction to modern convex geometry. In: Levy, S. (ed.) Flavors of Geometry. MSRI Publications, vol. 31, pp. 1-57. Cambridge University Press, Cambridge (1997)

3. Bastero, J., Romance, M.: John's decomposition of the identity in the non-convex case. Positivity 6 , $1-161(2002)$

4. Blumenthal, L.M., Wahlin, G.E.: On the spherical surface of smallest radius enclosing a bounded subset of $n$-dimensional Euclidean space. Bull. Am. Math. Soc. 47, 771-777 (1941)

5. Bourgain, J., Szarek, S.J.: The Banach-Mazur distance to the cube and the Dvoretzky-Rogers factorization. Isr. J. Math. 62, 169-180 (1988)

6. Dvoretzky, A., Rogers, C.A.: Absolute and unconditional convergence in normed linear spaces. Proc. Natl. Acad. Sci. USA 36, 192-197 (1950)

7. Giannopoulos, A.A.: A note on the Banach-Mazur distance to the cube. In: Lindenstrauss, J., Milman, V.D. (eds.) Geometric Aspects of Functional Analysis. Operator Theory: Advances and Applications, vol. 77, pp. 67-73. Birkhäuser, Basel (1995)

8. Giannopoulos, A.A., Milman, V.D.: Euclidean structure in finite-dimensional normed spaces. In: Johnson, W.B., Lindenstrauss, J. (eds.) Handbook on the Geometry of Banach Spaces, vol. 1, pp. 707-779. Elsevier, Amsterdam (2001)

9. Giannopoulos, A.A., Perissinaki, I., Tsolomitis, A.: John's theorem for an arbitrary pair of convex bodies. Geom. Dedic. 84, 63-79 (2001)

10. Gordon, Y., Litvak, A.E., Meyer, M., Pajor, A.: John's decomposition in the general case and applications. J. Differ. Geom. 68, 99-119 (2004)

11. Grünbaum, B.: Measures of symmetry for convex sets. AMS Proc. Symp. Pure Math. 7, 233-270 (1963)

12. John, F.: Extremum problems with inequalities as subsidiary conditions. In: Studies and Essays presented to R. Courant, pp. 187-204. Interscience, New York (1948)

13. Lassak, M.: Approximation of convex bodies by parallelotopes. Bull. Pol. Acad. Sci., Math. 39, 219$223(1991)$

14. Lemmens, P.W.H., Seidel, J.J.: Equiangular lines. J. Algebra 24, 494-512 (1973)

15. Sustik, M.A., Tropp, J.A., Dhillon, I.S. Jr., Heath, R.W.: On the existence of equiangular tight frames. Linear Algebra Appl. 426, 619-635 (2007)

16. Szarek, S.J.: Spaces with large distance to $\ell_{\infty}^{n}$ and random matrices. Am. J. Math. 112, 899-942 (1990) 
17. Szarek, S.J., Talagrand, M.: An isomorphic version of the Sauer-Shelah lemma and the BanachMazur distance to the cube. In: Lindenstrauss, J., Milman, V.D. (eds.) Geometric Aspects of Functional Analysis: Israel Seminar (GAFA) 1987-1988. Lecture Notes in Mathematics, vol. 1376, pp. 105-112. Springer, Berlin (1989)

18. Tomczak-Jaegermann, N.: Banach-Mazur Distances and Finite-Dimensional Operator Ideals. Pitman Monographs and Surveys in Pure and Applied Mathematics, vol. 38. Longman, Harlow (1989) 